\section{Microalbuminúria: Fator de Risco Cardiovascular e Renal Subestimado na Prática Clínica}

revisão

\author{
Maria Teresa Zanella
}

Embora de fácil determinação, a medida da excreção de albumina na urina tem sido pouco utilizada na identificação de indivíduos com diabetes tipo 2 (DM2), de maior risco para o desenvolvimento de doença renal e cardiovascular (CV). Tem sido demonstrado que as medidas das concentrações de albumina e creatinina $(\mathrm{Cr})$ em amostras isoladas de urina, permitindo o cálculo da relação entre elas, podem ser suficientes para o rastreamento e mesmo para a avaliação da eficácia de medidas adotadas para a redução da microalbuminúria. Valores $>30 \mathrm{mg} / \mathrm{g}$ de $\mathrm{Cr}$ ou $3,4 \mathrm{mg} / \mathrm{mmol}$ de $\mathrm{Cr}$ são indicativos de microalbuminúria e, em pacientes com DM2, a associação freqüente com a elevação dos níveis da pressão arterial representa condição de alto risco $\mathrm{CV}$. Evidências epidemiológicas indicam que a presença de microalbuminúria prediz maior morbidade e mortalidade CV independente de outros fatores de risco. Por outro lado, a microalbuminúria mostra-se também freqüentemente associada a outros fatores de risco $\mathrm{CV}$, sendo um dos componentes da síndrome metabólica. A capacidade de reduzir a pressão arterial, a pressão intraglomerular e a permeabilidade da membrana glomerular, fatores determinantes da progressão da lesão renal, explica o efeito renoprotetor dos inibidores da enzima conversora da angiotensina (IECAs) e dos bloqueadores dos receptores da angiotensina II (BRAs). No tratamento da nefropatia diabética, o uso de IECAs e BRAs associado ao controle rígido da pressão arterial, que deve ser mantida em níveis iguais ou inferiores a 130/80 mmHg, tem se mostrado como estratégia não só para promover proteção renal como também para promover proteção CV. (Arq Bras Endocrinol Metab 2006;50/2:313-321)

Descritores: Microalbuminúria; Risco cardiovascular; Proteção renal; Proteção cardiovascular

\section{ABSTRACT}

\section{Microalbuminuria: Cardiovascular and Renal Risk Factors} Underestimated in Clinical Practice.

Determination of microalbuminuria has been shown to be useful to identify patients with type 2 diabetes (DM2) at high risk of renal and cardiovascular (CV) diseases. The determination of the albumin/ creatinine $(\mathrm{Cr})$ ratio in an isolate sample of urine has been shown to be sufficient for the diagnosis as well as for the evaluation of the efficacy of the therapy employed to reduce microalbuminuria. Values of urinary albumin $>30 \mathrm{mg} / \mathrm{g}$ of $\mathrm{Cr}$ or $3,4 \mathrm{mg} / \mathrm{mmol}$ of $\mathrm{Cr}$ are evidence of microalbuminuria. This condition is frequently associated with high blood pressure levels, which increases dramatically not only the progression of renal disease but also de risk of a CV event. Epidemiologic studies have demonstrated that the presence of microalbuminuria is predictive of higher morbi-mortality independent of the presence of other CV risk factors. It appears to reflect a generalized vascular lesion not confined to the glomeruli. The capacity of reducing blood pressure, intraglomerular
Disciplina de Endocrinologia da UNIFESP, São Paulo, SP.
Recebido em 20/12/05 Aceito em 17/01/05 
pressure and the permeability of the glomerular membrane, which are important factors in the progression of renal disease, may explain the renoprotective effects of the angiotensin converting enzyme inhibitors (ACEIs) and the angiotensin II receptors blockers (ARBs). In the treatment of diabetic nephropathy, the control of blood pressure, which has to be maintained near or below 130/80 $\mathrm{mmHg}$ associated to the blockade of the reninangiotensin system with ACEls or BRAs are the best strategies to promote renal and CV protection. (Arq Bras Endocrinol Metab 2006;50/2:313-321)

Keywords: Microalbuminuria; Cardiovascular risk; Renal protection; Cardiovascular protection

A OCORRÊNCIA DE EXCREÇÃO URINÁRIA eXcessiva de albumina em indivíduos com diabetes tipo 2 é provavelmente o sinal de alerta mais precoce e importante do início de um comprometimento vascular generalizado (1), não somente limitado ao glomérulo, mas também presente em outros sítios vasculares, particularmente em órgãos vitais como o cérebro e o coração. Acredita-se que, sem uma intervenção específica, 20-40\% dos pacientes com diabetes tipo 2 e microalbuminúria progridem para a fase proteinúrica e finalmente para os estágios finais da doença renal $(2,3)$. Ainda que nos faltem evidências, a adoção de medidas terapêuticas que promovam a redução da microalbuminúria provavelmente se mostrará efetiva não só para promover proteção renal como também proteção cardiovascular. Assim sendo, a identificação e normalização da excreção urinária de albumina devem ser sempre consideradas no tratamento de pacientes com diabetes tipo 2 .

\section{Definição e determinação da microalbuminúria}

Embora de fácil de determinação, a medida da excreção de albumina na urina tem sido muito pouco utilizada como arma útil na identificação de indivíduos com diabetes tipo 2, passíveis de evoluírem para insufi- ciência renal crônica e de maior risco para o desenvolvimento de doença cardiovascular. A albuminúria pode ser definida através de diferentes medidas, como mostra a tabela 1 .

Existem basicamente três métodos diferentes de rastreamento para detectar aumentos na excreção urinária de albumina: 1 . Medida da relação entre as medidas de albumina e creatinina em amostra isolada de urina; 2. Medida da albuminúria em quantidade de urina obtida dentro de um período de tempo determinado (p. ex. 12 horas noturnas ou diurnas), expressa em microgramas por minuto $(\mu \mathrm{g} / \mathrm{min}) ; 3$. Determinação da quantidade de albumina excretada nas 24 horas, considerada como padrão ouro de determinação da albuminúria.

\section{Determinação em amostra isolada de urina}

A determinação da albuminúria em amostra isolada é a mais fácil de ser realizada na prática clínica e fornece informação bastante confiável. Tem sido demonstrado que este método tem uma sensibilidade de $90 \%$ na determinação da microalbuminúria comparada ao método de avaliação que utiliza a urina de 24 horas, mesmo após ajustes para idade e sexo $(4,5)$ A melhor avaliação é obtida quando a determinação é feita na primeira urina da manhã, evitando-se as variações diurnas que ocorrem com a atividade física. A ocorrência de microalbuminúria é pouco provável se, em uma amostra isolada de urina, a concentração de albumina for menor que 20 a $30 \mathrm{mg} / \mathrm{dl}$. Valores maiores, principalmente aqueles logo acima destes limites, podem representar resultados falso-positivos e devem ser confirmados repetindo-se as determinações. O problema de se medir apenas a concentração urinária de albumina é que podemos ter um número grande de resultados falso-positivos ou falsonegativos, uma vez que a concentração de albumina na urina é influenciada pelo volume urinário (6). Isto pode ser minimizado repetindo-se as determinações das quantidades de albumina na primeira urina da manhã (7).

Tabela 1. Valores da albuminúria utilizados para definições de micro- e macroalbuminúria em pacientes diabéticos.

\begin{tabular}{lccccc}
\hline & $\begin{array}{c}\text { Amostra } \\
\text { isolada } \\
\mathbf{m g} / \mathbf{m l}\end{array}$ & $\begin{array}{c}\text { Amostra } \\
\text { isolada } \\
\mathbf{m g} / \mathbf{g} \\
\text { Creatinina }\end{array}$ & $\begin{array}{c}\text { Amostra } \\
\mathbf{d e} \\
\mathbf{2 4 h s} \\
\mathbf{m g}\end{array}$ & $\begin{array}{c}\text { Amostra } \\
\text { de 12hs } \\
\text { diurnas } \\
\boldsymbol{\mu g} / \mathbf{m i n}\end{array}$ & $\begin{array}{c}\text { Amostra } \\
\text { de 12hs } \\
\text { noturnas } \\
\boldsymbol{\mu g} / \mathbf{m i n}\end{array}$ \\
\hline Normoalbuminúria & $<20$ & $<30$ & $<30$ & $<20$ & $<15$ \\
Microalbuminúria & $20-199$ & $30-299$ & $30-299$ & $20-199$ & $15-150$ \\
Macroalbuminúria & $\geq 200$ & $\geq 300$ & $\geq 300$ & $\geq 200$ & $\geq 150$ \\
\hline
\end{tabular}


Relação albumina / creatinina - O efeito do volume pode ser evitado totalmente se pudermos calcular a relação entre as concentrações de albumina e creatinina em uma amostra isolada de urina. Valores superiores a $30 \mathrm{mg} / \mathrm{g}$ de creatinina ou a $3,4 \mathrm{mg} / \mathrm{mmol}$ de creatinina levam a crer que a excreção de albumina nas 24 horas deve estar ultrapassando $30 \mathrm{mg} /$ dia e, portanto, são indicativas de microalbuminúria. Mesmo determinações em coleções de urina obtidas durante períodos de 12 ou 24 horas levam a resultados mais fidedignos se expressos através da relação albumina/ creatinina, uma vez que é difícil se fazer a coleta de forma perfeita. Perdas urinárias dentro de um determinado período subestimam a excreção urinária de albumina quando esta é expressa em $\mu \mathrm{g} / \mathrm{min}$. Isto nos faz concluir que as medidas das concentrações de albumina e creatinina em amostras isoladas, permitindo o cálculo da relação entre elas, podem ser suficientes para o rastreamento e mesmo para a avaliação da eficácia de medidas adotadas para a redução da microalbuminúria. Uma vez que existe uma certa variabilidade na excreção urinária de albumina, considera-se que duas ou três medidas dentro de um período de 3 a 6 meses devam se mostrar anormais, utilizando-se qualquer um dos métodos de coleta de urina para que se possa estabelecer o diagnóstico de microalbuminúria (2). Também é preciso ressaltar que parte da variabilidade nas medidas da albuminúria pode ser decorrente de exercícios, infecções ou febre no período prévio de 24 horas. Além disso, um controle inadequado dos níveis glicêmicos ou da pressão arterial, insuficiência cardíaca ou infecção urinária e até mesmo hematúria podem elevar a excreção de albumina acima dos valores normais (2). Por fim, é importante lembrar que deve ser sempre realizada a determinação das quantidades de albumina e não de proteína na urina. Medidas das concentrações de proteína na urina, quando em níveis baixos, não são sensíveis e são pouco confiáveis $(1,8)$.

\section{Microalbuminúria: evidência de lesão renal}

A presença de microalbuminúria no paciente diabético tipo 2 freqüentemente se associa à elevação dos níveis da pressão arterial, e representa uma condição de alto risco cardiovascular (9). Além de aumentos na pressão arterial sistêmica, dados clínicos e experimentais indicam que no diabetes ocorre aumento na pressão capilar glomerular. Esta é determinada não somente pela pressão arterial sistêmica, mas também por um distúrbio da auto-regulação renal e uma hemodinâmica glomerular alterada, caracterizada pela vasodilatação pré-glomerular e vasoconstrição pós-glomerular (10). A última é fortemente regulada pela atividade do sis- tema renina angiotensina intra-renal, que tem sua atividade aumentada pela hiperglicemia (11). O aumento da produção de angiotensina II no rim, através do estímulo do receptor $\mathrm{ATl}$, leva à menor produção de nefrina, proteína importante na integridade da membrana glomerular como barreira filtrante (12). A redução na produção de nefrina resulta em aumento da permeabilidade por aumento do tamanho dos poros da membrana glomerular. A pressão intraglomerular aumentada leva a hiperfiltração $\mathrm{e}$, na presença de um aumento da permeabilidade da membrana glomerular, favorece o aumento na excreção de albumina/proteína.

Além de aumentar a permeabilidade da membrana glomerular e de suas ações hemodinâmicas que favorecem a hipertensão intraglomerular, a AII também exerce efeitos nos rins, que contribuem para aumentar a produção da matriz extracelular (13). A hiperglicemia e a angiotensina II parecem produzir efeitos semelhantes sobre as células renais em cultura, estimulando a síntese da matriz protéica (14) e inibindo sua degradação enzimática (15).

Como demonstrado em estudos experimentais, a captação de proteínas filtradas pelas células epiteliais tubulares pode levar ao acúmulo anormal de proteínas nos endolisossomos e no retículo endoplasmático, que, por sua vez, podem estimular a produção de substâncias vasoativas e inflamatórias (16). Estas substâncias no interstício renal podem levar à proliferação de miofibroblastos, fibrogênese e fibrose. A ocorrência de hipertensão capilar glomerular, albuminúria e reabsorção de proteínas pelas células epiteliais tubulares pode ser a seqüência de eventos necessária para promover a lesão renal progressiva. A transudação de proteínas do plasma em outros leitos vasculares como o coração, por outro lado, poderia contribuir para o desenvolvimento de fibrose característica da cardiomiopatia diabética e para o maior risco de insuficiência cardíaca que se verifica em pacientes com macroproteinúria (16).

\section{Microalbuminúria: sinal de comprometimento vascular generalizado}

Evidências epidemiológicas indicam que a presença de microalbuminúria prediz maior morbidade e mortalidade cardiovascular independente de outros fatores de risco (1). Tem sido demonstrada uma correlação positiva e praticamente linear entre a presença de quantidades aumentadas de albumina na urina e a ocorrência de infarto do miocárdio ou de acidentes vasculares cerebrais, tanto em pacientes com diabetes tipo 2 quanto em pacientes não diabéticos (17-20). 
Gerstein e col., analisando os resultados do estudo HOPE (Heart Outcomes Prevention Evaluation), verificaram que, entre mais de 9.000 participantes considerados de alto risco cardiovascular, a microalbuminúria se mostrava como um forte fator preditivo dos principais eventos cardiovasculares (infarto do miocárdio, acidente vascular cerebral e morte cardiovascular) e mortalidade por todas as causas, tanto em pacientes diabéticos como em pacientes não diabéticos. Além disso, foi observada correlação linear entre valores da razão albumina/creatinina em amostra isolada e a morbi-mortalidade cardiovascular. No início do estudo, os valores da razão albumina/ creatinina mostravam-se elevados em 33\% dos pacientes diabéticos e $15 \%$ dos pacientes não diabéticos (21).

Foi também observado aumento do risco cardiovascular associado ao aumento da excreção urinária de albumina no estudo LIFE, que incluiu 7.143 pacientes hipertensos não diabéticos e 1.063 pacientes hipertensos com diabetes, todos apresentando evidências eletrocardiográficas de hipertrofia ventricular esquerda (22). Neste estudo, para cada aumento de 10 vezes no valor da relação albumina/creatinina, o risco de morte por doença cardiovascular, infarto do miocárdio ou acidente vascular cerebral aumentava em $57 \%$ em pacientes não diabéticos e $39 \%$ em pacientes diabéticos.

\section{Microalbuminúria e síndrome metabólica}

A microalbuminúria mostra-se também freqüentemente associada a outros fatores de risco cardiovascular (23), fazendo parte da condição que conhecemos como síndrome metabólica. Esta se caracteriza pela constelação de fatores de risco cardiovascular que inclui hipertensão arterial, obesidade central, dislipidemia, intolerância à glicose, um estado de hipercoagulabilidade, alem de lesão endotelial que se manifesta através da microalbuminúria.

Existem evidências de que aproximadamente $40 \%$ dos pacientes diabéticos tipo 2 com microalbuminúria, em decorrência de lesão vascular progressiva, evoluirão ao longo de uma década para a condição de macroproteinúria (24-26). Esta condição, que revela lesão vascular mais grave, associa-se a um risco de eventos cardiovasculares ainda maior (17).

Se considerarmos a vasta interface sangue-filtrado glomerular provida por 500.000 a 1.000 .000 de glomérulos renais, fica fácil compreender porque um sinal de lesão na superfície vascular envolvida na filtração glomerular pode representar medida fidedigna de comprometimento vascular generalizado por todo o organismo. A excreção aumentada de albumina ou proteína na urina de pacientes diabéticos seria resultado de um distúrbio mais abrangente da função de barreira da célula endotelial, ocorrendo também na macrocirculação, especialmente nas coronárias, onde a transudação da proteína do plasma para a parede do vaso poderia promover o processo aterogênico (27). Assim, a microalbuminúria, parâmetro de avaliação da microcirculação renal, pode ser utilizada também como parâmetro de lesão vascular generalizada, não confinada aos glomérulos. Nesta condição, existe, portanto, a necessidade de maior atenção para as medidas de controle dos diversos fatores de risco cardiovascular, em particular da hipertensão arterial, objetivando atingir com mais rigor as metas desse controle e a proteção vascular. Embora as diretrizes preconizem que valores da pressão arterial inferiores a $130 / 80 \mathrm{mmHg}$ devam ser atingidos para maior proteção renal e cardiovascular $(28,29)$ em pacientes diabéticos, é possível que níveis até mais baixos precisem ser atingidos quando se constata a presença de microalbuminúria.

Resultados de estudos mais recentes mostram que mesmo valores baixos da albuminúria, bem inferiores àqueles que atualmente definem a microalbuminúria, tem sido associados a um aumento do risco cardiovascular, fazendo crer que os limites da normalidade devam ser revistos.

No Third Copenhagen City Heart Study (30) foram incluídos, entre 1992 e 1994, 1.734 pacientes hipertensos sem história de doença coronariana, que foram submetidos a coleta de urina para a medida da albuminúria durante 12 horas noturnas, e seguidos até 2000 no que se referia ao desenvolvimento de doença coronariana e até 2004 no que se referia à mortalidade.

Durante o seguimento, 123 pacientes apresentaram evidências de doença coronariana e foram constatadas 309 mortes. A incidência de doença coronariana foi de $11 \%$ em indivíduos com excreção urinária de albumina $\geq 5 \mu \mathrm{g} / \mathrm{min}$ e de $5 \%$ em indivíduos com excreção urinária de albumina $<5 \mu \mathrm{g} / \mathrm{min}(\mathrm{p}<0,001)$.

Da mesma forma, a mortalidade acumulada no período foi de $28 \%$ e $13 \%$ ( $\mathrm{p}<0,001)$ nos indivíduos com excreção urinária de albumina maior e menor que $5 \mathrm{mg} / \mathrm{min}$ respectivamente. Os riscos relativos de doença cardíaca coronariana e morte associada à excreção urinária de albumina $\geq 5 \mu \mathrm{g} / \mathrm{min}$ foram de $2,0(1,4$ a 2,$9 ; \mathrm{p}<0,001)$ e $1,9(1,5$ a 2,$3 ; \mathrm{p}<0,001)$, respectivamente.

Também foi observado maior risco de desenvolvimento de hipertensão arterial associado a níveis baixos de albuminúria em 1.499 indivíduos não diabéticos normotensos seguidos por 2,9 anos no Fra- 
mingham Heart Study (31). Em análise multivariada ajustada para os conhecidos fatores de risco, a razão da chance de desenvolvimento de hipertensão foi de 1,20 para cada desvio padrão da relação albumina/creatinina. Os indivíduos que apresentavam valores da relação albumina/creatinina no quintil superior $(6,6 \mathrm{mg} / \mathrm{g}$ nos homens, $15,4 \mathrm{mg} / \mathrm{g}$ nas mulheres) em relação àqueles com valores no quintil mais inferior, apresentaram uma probabilidade 1,93 maior de desenvolver hipertensão.

\section{Hipertensão arterial}

A pressão arterial elevada é um fenômeno precoce e freqüente na nefropatia diabética, e tem sido documentada uma relação direta entre a pressão arterial e a taxa de declínio de TFG em pacientes com diabetes tipos 1 e $2(32,33)$. Vários estudos mostram claramente que a terapia anti-hipertensiva precoce e intensiva reduz com eficácia tanto a albuminúria quanto a taxa de declínio da TFG em pacientes com nefropatia diabética clinicamente manifesta $(34,35)$.

Contrariamente ao que ocorre no diabetes tipo 1 , nos pacientes com diabetes tipo 2 , a hipertensão não necessariamente está vinculada à presença de doença renal e, em geral, precede o diagnóstico de diabetes. A hipertensão é duas vezes mais freqüente em pacientes diabéticos que em não diabéticos e está fortemente relacionada ao aumento do peso corporal, resistência à insulina e hiperinsulinemia (36).

A hipertensão aumenta o risco de morbidade e mortalidade cardiovascular, especialmente quando a função renal começa a decair $(10,25,26)$. A combinação de diabetes e hipertensão está associada a um aumento de cerca de 4 vezes no risco cardiovascular, se comparada à população diabética normotensa. Adler e cols. avaliaram o risco de complicações diabéticas associadas à pressão arterial sistólica (PAS) em 3.642 pacientes com diabetes tipo 2 recém diagnosticados, entre 25 e 65 anos de idade, inscritos no UKPDS (37).

Os pacientes foram acompanhados por cerca de 10,4 anos para avaliação da mortalidade por todas as causas, infarto do miocárdio, insuficiência cardíaca, AVC, amputação da extremidade inferior ou complicações microvasculares. O aumento da PAS foi significativamente associado tanto à mortalidade quanto ao desenvolvimento das complicações $(\mathrm{p}<0,0001)$. O aumento de risco à medida que se eleva a PAS se mostrou contínuo e linear, sugerindo a inexistência de um ponto de corte para a ocorrência de qualquer complicação decorrente do diabetes ou para morte.

Sendo a hipertensão um dos principais fatores que contribuem para as complicações do diabetes, o controle dos níveis pressóricos é essencial na terapia de pacientes com nefropatia diabética. Dados obtidos no UKPDS (38) e no HOT Study (39) mostraram que o controle da pressão arterial de forma intensiva não só reduz as complicações e a mortalidade no diabetes tipo 2, mas também que não existe um limite inferior da pressão arterial estabelecido abaixo do qual não se deva prosseguir com o tratamento. Quanto mais baixos os níveis pressóricos, menores os riscos de complicações

\section{Angiotensina II (AlI) e nefropatia diabética}

Apesar da atividade plasmática de renina encontrar-se reduzida em pacientes diabéticos (40), estudos múltiplos em modelos animais mostram que os níveis teciduais de renina e mesmo a produção de renina são normais ou até mesmo elevados (41). Portanto, as medidas da atividade da renina ou mesmo da AII na circulação podem ser enganosas, fornecendo poucas informações sobre o estado do sistema renina-angiotensina intra-renal. Vale lembrar que a AII é a mediadora principal do SRA e parece ser responsável por importantes efeitos patológicos em nível celular por meio de estimulação do receptor ATl da AII.

Os benefícios dos bloqueadores do sistema renina-angiotensina na nefropatia diabética são vistos como resultado, pelo menos em parte, da atenuação dos efeitos da AII na pressão arterial e na hemodinâmica glomerular (42). Evidências indiretas indicam que a acentuada resposta vasodilatadora renal aos inibidores da enzima conversora da angiotensina (IECAs) e aos bloqueadores dos receptores da AII (BRAs) em pacientes diabéticos pode ser atribuída à redução dos efeitos da AII no rim. Ao reduzir os efeitos vasoconstritores da AII na arteríola glomerular eferente, os IECAs reduzem a pressão intraglomerular, a qual parece ser um determinante importante da progressão de nefropatia diabética.

Em indivíduos saudáveis e pacientes hipertensos, os BRAs possuem efeitos idênticos aos dos IECAs na hemodinâmica renal, decorrente de sua ação direta sobre os receptores de ATl (43). Também tem sido demonstrado que os BRAs são capazes de reduzir o tamanho dos poros excessivamente grandes da membrana glomerular, um mecanismo também envolvido no efeito antiproteinúrico destes agentes. Experimentalmente, já se demonstrou que o efeito antiproteinúrico do irbesartana se associa ao aumento da expressão glomerular da nefrina, que reduz a permeabilidade da membrana glomerular comprometida (44).

A AII também exerce, nos rins, efeitos que podem contribuir para aumentar a produção da matriz extracelular, estimulando a síntese da TGF- $\beta 1$ por 
meio da ativação do seu receptor ATl (45). A AII também ativa a proteína quinase $\mathrm{C}$ (PK-C) nas células renais tubulares proximais e mesangiais por meio dos receptores ATl (46). Esses efeitos não-hemodinâmicos da AII são muito semelhantes aos da hiperglicemia e existem evidências, obtidas in vitro e in vivo, de que a hiperglicemia ativa o sistema renina-angiotensina tecidual $(12,47)$.

\section{Microalbuminúria: importância do bloqueio do SRA}

A capacidade de reduzir a pressão intraglomerular e a permeabilidade da membrana glomerular explica o efeito antiproteinúrico dos IECAs e BRAs demonstrado em estudos randomizados prospectivos de desfecho, os quais documentaram menor velocidade de deterioração da função renal durante o bloqueio do SRA.

O uso de IECAs e BRAs tem se mostrado nitidamente benéfico, não só para promover proteção renal em pacientes com nefropatia diabética como também para promover proteção cardiovascular em pacientes diabéticos com outros fatores de risco $(20,21)$. Com relação à proteção renal, a maioria dos resultados com IECAS foi obtida em pacientes com diabetes tipo 1. Resultados mais recentes foram obtidos com BRAs em pacientes diabéticos do tipo 2 .

Os estudos RENAAL (48) (The Reduction in Renal Endpoints in Type 2 Diabetes with Angiotensin II Antagonist Losartan) e IDNT (49) (Irbesartan Diabetic Nephropathy Trials) foram realizados $\mathrm{em}$ pacientes com diabetes tipo 2, hipertensos, com insuficiência renal inicial e proteinúria clínica. $\mathrm{O}$ resultados mostraram claramente que o bloqueio dos receptores ATl da AII, quer com 100 mg de losartan, quer com $300 \mathrm{mg}$ de irbesartana, mostrou-se como a estratégia mais eficiente para prevenir o desfecho composto, que incluía duplicação nos níveis séricos de creatinina, insuficiência renal terminal ou morte, quando comparada à abordagem terapêutica tradicional que compreendia o uso de medicação anti-hipertensiva sem o emprego de bloqueadores do sistema renina-angiotensina.

A importância de se reduzir a excreção urinária de proteínas foi demonstrada no estudo IDNT, onde a proteinúria inicial foi capaz de prever a progressão para os desfechos do estudo, mostrando um risco dobrado para cada duplicação nos valores da proteína urinária. Além disso, redução de $50 \%$ na proteinúria observada aos 12 meses de tratamento com irbesartana se associou a uma redução de $50 \%$ no risco de duplicação da creatinina ou de atingir os estágios finais da insuficiência renal.
Embora o uso de bloqueadores do sistema renina-angiotensina seja importante na prevenção da nefropatia diabética, é importante lembrar que o controle da pressão arterial é essencial e que a maioria dos pacientes requer a combinação de múltiplas drogas para reduzi-la a níveis que permitam a correção da hipertensão no capilar glomerular e a redução máxima da proteinúria. Embora se preconize que em pacientes diabéticos devam ser atingidos valores da pressão arterial abaixo de $130 / 80 \mathrm{mmHg}$, é possível que a manutenção de níveis ainda mais baixos possa trazer maiores benefícios.

Talvez se deva procurar atingir níveis de pressão arterial suficientes para reduzir a microalbuminúria em pelo menos $50 \%$, incluindo sempre, entre os antihipertensivos utilizados, bloqueadores do sistema renina-angiotensina em doses máximas. No estudo IRMA2 (50), no grupo de pacientes diabéticos tipo 2 microalbuminúricos em tratamento com $300 \mathrm{mg} /$ dia de irbesartana, uma redução de $46 \%$ na albuminúria se associou, após dois anos, à menor progressão da lesão renal para a fase proteinúrica, quando comparada à elevação de $9 \%$ observada no grupo controle, no qual a medicação anti-hipertensiva não incluía bloqueadores do SRA ( $5,2 \%$ vs. $14,9 \%$, respectivamente). Este efeito se mostrou independente da ação antihipertensiva da droga, uma vez que os três grupos estudados, tratados com placebo ou irbesartana nas doses de 150 e $300 \mathrm{mg}$, atingiram níveis semelhantes da pressão arterial. Além disso, no grupo de pacientes em uso de $300 \mathrm{mg}$ de irbesartana, $34 \%$ dos pacientes microalbuminúricos tornaram-se normoalbuminúricos, o que ocorreu em apenas $21 \%$ dos pacientes em uso de placebo $(\mathrm{p}<0,0006)$.

Mais recentemente, demonstrou-se que a utilização de $900 \mathrm{mg}$ de irbesartana em dose única diária em pacientes diabéticos tipo 2 com microalbuminúria promove um bloqueio ainda mais completo do sistema renina-angiotensina e uma redução adicional na excreção urinária de albumina, independente de alterações nos níveis pressóricos e na filtração glomerular (51).

Os pacientes que apresentavam os valores mais elevados da albuminúria com a dose de $300 \mathrm{mg}$ foram os que mais se beneficiaram com o uso da dose de 900 mg. Estudos adicionais são necessários para se verificar se existem benefícios da utilização desta dose elevada também na fase proteinúrica da nefropatia diabética, e se um efeito antiproteinúrico maior pode ser obtido com doses ainda mais elevadas de irbesartana, uma vez que um platô na curva de dose-resposta não foi atingido, apesar da dose de $900 \mathrm{mg}$ ser muito superior à usualmente recomendada $(51)$. 
Com relação à utilização dos inibidores da ECA em pacientes com diabetes tipo 2 e microalbuminúria ou proteinúria instalada, o único estudo aleatório comparou o enalapril ao BRA, a telmisartana, no ensaio DETAIL, que incluiu 250 pacientes com nefropatia inicial, definida pelo valor da albuminúria $(82 \% \mathrm{com}$ microalbuminúria e $18 \%$ macroalbuminúria de no máximo de 1,4 g/dia) e uma taxa de filtração glomerular (TFG) basal (medida com a utilização de radioisótopo) de cerca de $93 \mathrm{ml} / \mathrm{min}$ por $1,73 \mathrm{~m}^{2}$ (52).

Uma queda na TFG mais acentuada, próxima a $10,0 \mathrm{~mL} / \mathrm{min}$ por $1,73 \mathrm{~m}^{2}$, após cinco anos, foi prédefinida como indicador de uma diferença clinicamente significativa entre os grupos. Após cinco anos, houve uma queda mais atenuada na TFG do grupo enalapril, não significativa estatisticamente $(14,9$ versus $17,9 \mathrm{~mL} / \mathrm{min}$ por $1,73 \mathrm{~m}^{2}$ no grupo telmisartana). Ambos os grupos se mostraram semelhantes para os desfechos secundários, os quais incluíram alterações anuais na TFG, pressão arterial, creatinina sérica, albumina urinária, doença renal crônica terminal, eventos cardiovasculares e mortalidade.

As duas limitações do estudo foram as de que somente 168 dos 250 pacientes concluíram o estudo e de que somente $20 \%$ apresentaram macroalbuminúria. Embora tenha sido feita apenas esta única comparação entre as duas classes de bloqueadores do sistema renina-angiotensina, os resultados condizem com a hipótese de que os inibidores de ECA seriam pelo menos tão eficazes quanto os BRAs em pacientes diabéticos com microalbuminúria.

\section{Combinação de um IECA com um BRA}

Discute-se se haveria vantagens na associação de um IECA com um BRA. Em um estudo clínico controlado que incluiu pacientes diabéticos do tipo $2 \mathrm{com}$ microalbuminúria, o lisinopril $(20 \mathrm{mg} / \mathrm{dia})$ e a candersartana $(16 \mathrm{mg} / \mathrm{dia})$ produziram reduções equivalentes na pressão arterial, mas que se mostraram inferiores àquela obtida com a terapia combinada $(14,1 / 10,7$ e $16,7 / 10,4$ $\mathrm{mmHg}$ versus $25,3 / 16,3 \mathrm{mmHg}$ ) (53). A candesartana também provocou redução da albuminúria, mas este efeito se mostrou menor que o obtido com o lisinopril ou com a terapia combinada. Entretanto, a combinação dos dois agentes não foi comparada a doses máximas de cada um dos agentes administrados isoladamente. É possível que doses maiores de cada um deles isoladamente resultassem em reduções equivalentes ou até maiores daquela obtida com a combinação. De fato, com relação à irbesartana, já se demonstrou que o aumento da dose utilizada promove reduções adicionais na excreção urinária de albumina (51).

\section{Recomendações}

A detecção da microalbuminúria é medida importante para identificar indivíduos com maior risco de doença cardiovascular e com lesão renal passível de progressão para a insuficiência renal. $\mathrm{O}$ rastreamento para microalbuminúria, segundo as recomendações da American Diabetes Association e da Sociedade Brasileira de Diabetes, deve ser feito anualmente em pacientes diabéticos do tipo 1 a partir do quinto ano do diagnóstico e em pacientes com diabetes do tipo 2 a partir do diagnóstico. A presença de microalbuminúria requer maior atenção para as medidas de controle da pressão arterial, que deve ser mantida em níveis inferiores a $130 / 80 \mathrm{mmHg}$, assim como para as medidas de controle dos lípides e glicemia, além da adoção de medidas eficazes na redução da excreção urinária de albumina como o uso de inibidores da ECA ou de antagonistas da angiotensina II. Estes últimos, em pacientes diabéticos do tipo 2 já na fase proteinúrica da nefropatia diabética, se mostraram comprovadamente eficazes para promover renoproteção e certo grau de cardioproteção, uma vez que também reduzirão a necessidade de hospitalizações por insuficiência cardíaca $(48,49)$.

\section{REFERÊNCIAS}

1. Keane WF, Eknoyan G. Proteinuria, albuminuria, risk, assessment, detection, elimination (PARADE): a position paper of the National Kidney Foundation. Am J Kidney Dis 1999;33:1004-10.

2. American Diabetes Association. Standards of medical care for patients with diabetes mellitus. Diabetes Care 2003:26(suppl 1):S33-S50.

3. Harris MI, Flegal KM, Cowie CC, Eberhardt MS, Goldstein $D E$, Little RR, et al. Prevalence of diabetes, impaired fasting glucose, and impaired glucose tolerance in US adults: the Third National Health and Nutrition Examination Survey, 1988-1994. Diabetes Care 1998;21:518-24.

4. Bennett PH, Haffner S, Kasiske BL, Keane WF, Mogensen $\mathrm{CE}$, Parving $\mathrm{HH}$, et al. Screening and management of microalbuminuria in patients with diabetes mellitus: recommendations to the Scientific Advisory Board of the National Kidney Foundation from an ad hoc committee of the Council on Diabetes Mellitus of the National Kidney Foundation. Am J Kidney Dis 1995:25:107-12.

5. Houlihan CA, Tsalamandris C, Akdeniz A, Jerums G. Albumin to creatinine ratio: a screening test with limitations. Am J Kidney Dis 2002;39:1 183-9.

6. Schwab SJ, Dunn FL, Feinglos MN. Screening for microalbuminuria. A comparison of single sample methods of collection and techniques of albumin analysis. Diabetes Care 1992;15:1581. 
7. Mogensen $\mathrm{CE}$, Vestbo $\mathrm{E}$, Poulsen $\mathrm{PL}$, Christiansen C, Damsgaard EM, Eiskjaer $\mathrm{H}$, et al. Microalbuminuria and potential confounders. A review and some observations on variability of urinary albumin excretion. Diabetes Care 1995; 18:572-81.

8. Pegoraro AA, Peracha W, Hasnain M, Ranginwala N, Shaykh $M$, singh AK, et al. Evaluation of a new fluorescent dye method to measure urinary albumin in lieu of urinary total protein. Am J Kidney Dis 2000;35:739-44.

9. Valmadrid C, Klein R, Moss SE, Klein BE. The risk of cardiovascular mortality associated with microalbuminuria and gross proteinuria in persons with older onset diabetes mellitus. Arch Intern Med 2000; 160:1093-8.

10. Amos AF, McCarty DJ, Zimmet $P$. The rising global burden of diabetes and its complications; estimates and projections to the year 2010. Diabet Med (England) 1997; 14(suppl 5):S1-S85.

11. Hollenberg NK. ACE inhibition, angiotensin II receptor blockade and diabetic nephropathy. In: Mogensen CE, editor. The kidney and hypertension in diabetes mellitus. Boston:Kluwer Academic Publishers, 2000. p.417-22.

12. Langham RG, Kelly DJ, Cox AJ, Thomson NM, Holthofer $H$, Zaoui $P$, et al. Proteinuria and the expression of the podocyte slit diaphragm protein, nephrin, in diabetic nephropathy: effects of angiotensin converting enzyme inhibition. Diabetologia 2002;45:1572-6.

13. Wolf $G$. Angiotensin II is involved in the progression of renal disease: importance of non-hemodynamic mechanisms. Nephrologie 1998; 19:451-6.

14. Ayo SH, Radnik RA, Garoni J, Glass II WF, Kreisberg JI. High glucose causes an increase in extracellular matrix proteins in cultured mesangial cells. Am J Pathol 1990; 136: 1339-48.

15. McLennan SV, Fisher EJ, Yue DK, Turtle JR. High glucose concentration causes a decrease in mesangium degradation: a factor in the pathogenesis of diabetic nephropathy. Diabetes 1994;43:1041-5.

16. Miettinen H, Haffner SM, Lehto S, Ronnemaa T, Pyorala K, Laakso M. Proteinuria predicts stroke and other atherosclerotic vascular disease events in nondiabetic and non-insulin-dependent diabetic subjects. Stroke 1996;27:2033-9.

17. Hockensmith ML, Estacio RO, Mehler P, Havranek EP, Ecder ST, Lundgren RA, et al. Albuminuria as a predictor of heart failure hospitalizations in patients with type 2 diabetes. J Card Fail 2004; 10:126-31.

18. Schmitz A, Vaeth M. Microalbuminuria: a major risk factor in non-insulin-dependent diabetes. A 10-year follow-up study of 503 patients. Diabet Med 1988:5:126-34.

19. Tuttle KR, Puhlman ME, Cooney SK, Short R. Urinary albumin and insulin as predictors of coronary artery disease: an angiographic study. Am J Kidney Dis 1999:34:918-25.

20. Borch-Johnsen K, Feldt-Rasmussen B, Strandgaard S, Schroll M, Jensen JS. Urinary albumin excretion. An independent predictor of ischemic heart disease. Arterioscler Thromb Vasc Biol 1999;19:1992-7.

21. Gerstein HC, Mann JF, Yi Q, Zinman B, Dinneen SF, Hoogwerf B, et al. Albuminuria and risk of cardiovascular events, death, and heart failure in diabetic and nondiabetic individuals. JAMA 2001;286:421-6.
22. Wachtell K, Ibsen $H$, Olsen MH, Borch-Johnsen K, Lindholm LH, Mogensen CE, et al. Albuminuria and cardiovascular risk in hypertensive patients with left ventricular hypertrophy: the LIFE study. Ann Intern Med 2003: 139:901-6.

23. Sowers JR, Epstein M, Frohlich ED. Diabetes, hypertension, and cardiovascular disease: an update. Hypertension 2001;37:1053-9.

24. Mogensen CE. Microalbuminuria predicts clinical proteinuria and early mortality in maturity onset diabetes. N Engl J Med 1984;310:356-60.

25. Esmail ZN, Loewen PS. Losartan as an alternative to ACE inhibitors in patients with renal dysfunction. Ann Pharmacother 1998:32:1096-8.

26. Parving $\mathrm{HH}$. Diabetic nephropathy: prevention and treatment. Kidney Int 2001;60:2041-55.

27. Deckert T. Nephropathy and coronary death. The fatal twins in diabetes mellitus. Nephrol Dial Transplant 1994:9:1069-71.

28. Chobanian AV, Bakris GL, Black HR, Cushman WC. The Seventh Report of the Joint National Committee on Prevention, Detection, Evaluation, and Treatment of High Blood Pressure: The JNC 7 Report. Hypertension 2003;42:1206-52.

29. Bakris GL, Williams M, Dworkin L, Elliott WJ, Epstein M, Toto $\mathrm{R}$, et al. Preserving renal function in adults with hypertension and diabetes: a consensus approach. National Kidney Foundation Hypertension and Diabetes Executive Committees Working Group. Am J Kidney Dis 2000;36:646-61.

30. Klausen KP, Scharling $H$, Jensen $G$, Jensen JS. New definition of microalbuminuria in hypertensive subjects: association with incident coronary heart disease and death. Hypertension 2005;46:33-7.

31. Wang TJ, Evans JC, Meigs JB, Rifai N, Fox CS, D'Agostino $R B$, et al. Low-grade albuminuria and the risks of hypertension and blood pressure progression. Circulation 2005; 111 :1370-6.

32. Yokoyama $\mathrm{H}$, Tomonaga $\mathrm{O}$, Hirayama $\mathrm{M}$, Ishii $\mathrm{A}$, Takeda $\mathrm{M}$, Babazono $\mathrm{T}$, et al. Predictors of the progression of diabetic nephropathy and the beneficial effect of angiotensin converting enzyme inhibitors in NIDDM patients. Diabetologia 1997:40:405-11.

33. Parving HH, Hommel E, Mathiesen ER. Prevalence of microalbuminuria, arterial hypertension, retinopathy and neuropathy in patients with insulin dependent diabetes. BMJ 1988;296:156-60.

34. Parving HH, Andersen AR, Smidt UM, Svendsen PA. Early aggressive antihypertensive treatment reduces rate of decline in kidney function in diabetic nephropathy. Lancet 1983;1:1175-9.

35. Parving $\mathrm{HH}$, Smidt UM, Hommel E, Mathiesen ER, Rossing $P$, Nielsen $F$, et al. Effective antihypertensive treatment postpones renal insufficiency in diabetic nephropathy. Am J Kidney Dis 1993:22:188-95.

36. Hypertension in Diabetes Study (HDS). I- Prevalence of hypertension in newly presenting type 2 diabetic patients and the association with risk factors for cardiovascular and diabetic complications; II. Increased risk of cardiovascular complications in hypertensive type-2 diabetic patients. J Hypertens 1993: 11:307-17. 
37. Adler A, Stratton IM, Neil HA, Yudkin JS, Matthews DR, Cull CA, et al. Association of systolic blood pressure with macrovascular and microvascular complications of type 2 diabetes (UKPDS 36): prospective observational study. BMJ 2000;321:412-9.

38. UK prospective Diabetes Study Group. Tight blood pressure control and risk of microvascular and macrovascular complications in type 2 diabetes: UKPDS 38. BMJ 1998;317:703-13.

39. Hansson L, Zanchetti A, Carruthers SG, Dahlof B, Elmfeldt $D$, Julius $S$, et al. Effects of intensive blood pressure lowering and low dose aspirin in patients with hypertension: principle results of the Hypertension Optimal Treatment (HOT) randomized trial. Lancet 1998;351:1755-62.

40. Björk S. The renin angiotensin system in diabetes mellitus: a physiological and therapeutic study. Scand J Urol Nephrol 1990; 126(suppl): 1-50.

41. Anderson S, Jung FF, Ingelfinger JR. Renal reninangiotensin system in diabetes: functional immunohistochemical and molecular biological correlations. Am J Physiol 1993;265:F477-86.

42. Zatz R, Dunn BR, Meyer TW, Anderson S, RennKe GH, Brenner BM. Prevention of diabetic glomerulopathy by pharmacological amelioration of glomerular capillary hypertension. J Clin Invest 1986;77:1925-30.

43. Deray G. Do antagonists of angiotensin II ATI receptors protect the kidney? Arch Mal Coeur Vaiss 1999;92:903-7.

44. Bonnet F, Cooper ME, Kawachi H, Allen TJ, Boner G, Cao Z. Irbesartan normalizes the deficiency in glomerular nephrin expression in a model of diabetes and hypertension. Diabetologia 2001;44:874-7.

45. Kagami S, Border WA, Miller DE, Noble NA. Angiotensin II stimulates extracellular matrix protein synthesis through induction of transforming growth factor-beta expression in the rat glomerular mesangial cells. J Clin Invest 1994:93:2431-7.

46. Wolf $\mathrm{G}$, Ziyadeh $\mathrm{FN}$. The role of angiotensin II in diabetic nephropathy: emphasis on non-hemodynamic mechanisms. Am J Kidney Dis 1997;29:153-63.
47. Singh R, Alavi $N$, Singh AK, Leehey DJ. Role of angiotensin II in glucose induced inhibition of mesangial matrix degradation. Diabetes 1999:48:2066-73.

48. Brenner BM, Cooper ME, de Zeeuw D, Keane WF, Mitch WE, Parving $\mathrm{HH}$, et al; the RENAAL Study Investigators. Effects of losartan on renal and cardiovascular outcomes in patients with type 2 diabetes and nephropathy. N Eng J Med 2001;345:861-9.

49. Lewis EJ, Hunsicker LG, Clarke WR, Berl T, Pohl MA, Lewis $\mathrm{JB}$, et al; The Collaborative Study Group. Renoprotective effect of the angiotensin receptor antagonist irbersartan in patients with nephropathy due to type 2 diabetes. N Eng J Med 2001;345:851-60.

50. Parving $\mathrm{HH}$, Lehnert $\mathrm{H}$, Bröchner-Mortensen J, Gomis R, Andersen R, Arner P; the Irbersartan in Patients with Type 2 Diabetes and Microalbuminuria Study Group. The effect of irbersartan on the development of diabetic nephropathy in patients with type 2 diabetes. $\mathbf{N}$ Eng J Med 2001;345:870-8.

51. Rossing K, Schjoedt KJ, Jensen BR, Boomsma F, Parving $\mathrm{HH}$. Enhanced renoprotective effects of ultrahigh doses of irbesartan in patients with type 2 diabetes and microalbuminuria Kidney Int 2005;68: 1 190-8.

52. Barnett AH, Bain SC, Bouter P, Karlberg B, Madsbad S, Jervell $\mathrm{J}$, et al; Diabetics Exposed to Telmisartan and Enalapril Study Group. Angiotensin-receptor blockade versus converting-enzyme inhibition in type 2 diabetes and nephropathy. N Engl J Med 2004;351:1952-61.

53. Mogensen CE, Neldam S, Tikkanen I. Randomised controlled trial of dual blockade of renin-angiotensin system in patients with hypertension, microalbuminuria, and non-insulin dependent diabetes: the candesartan and lisinopril microalbuminuria (CALM) study. BMJ 2000;321:1440-4.

\section{Endereço para correspondência:}

Maria Teresa Zanella

Rua Leandro Dupret 365

04025-002 São Paulo, SP

E-mail: tereza.zanella@hrim.com.br 